\title{
Comments to 'Vortex clustering, polarisation and circulation intermittency in classical and quantum turbulence'
}

Zambri Harun ${ }^{1}$

1 Universiti Kebangsaan Malaysia

Potential competing interests: The author(s) declared that no potential competing interests exist.

\section{Comments}

Abstract

Introduction

It would be great if Figure 1 is accompanied by some kind of scale so that readers can get some sense of geometry e.g. how big is the eddies / filament.

While describing Figure 2, the authors need to define the wave number $k$ and lambda so that readers will not be left thinking what they are.

\section{Results}

It would be nice if there is a discussion the difference in energy distribution in quantum turbulence as compared with the conventional turbulent fow. 